\title{
ESTRATEGIAS INTRAHOSPITALARIAS DE MEJORA DEL TIEMPO PUERTA-BALÓN Y EVOLUCIÓN CLÍNICA EN PACIENTES CON INFARTO AGUDO DE MIOCARDIO CON SUPRADESNIVEL DEL ST
}

\author{
INTRAHOSPITAL STRATEGIES FOR IMPROVING DOOR-TO-BALLOON \\ TIME AND CLINICAL EVOLUTION IN PATIENTS WITH ACUTE \\ MYOCARDIAL INFARCTION WITH ST-SEGMENT ELEVATION
}

\author{
ALAN SIGAL ${ }^{1}$, ANDREÍNA GIL RAMÍREZ' ${ }^{1}$ CRISTIAN GARMENDIA ${ }^{1}$, NOELIA ALBY ${ }^{1}$, LUCRECIA M. BURGOS², FERNANDO CURA ${ }^{3}$, \\ ALFONSINA CANDIELLO ${ }^{4}$
}

\begin{abstract}
RESUMEN
Introducción. El tratamiento exitoso del infarto con supradesnivel del ST (IAMCEST) es tiempo dependiente. Existe limitada información en nuestro país acerca de la efectividad de estrategias en la reducción de los tiempos de isquemia. E objetivo de este trabajo es analizar el impacto de la aplicación de estrategias intrahospitalarias de mejora (EIHM) en la reducción del tiempo puerta balón (TPB), y en la evolución clínica de pacientes con IAMCEST.

Métodos. Estudio observacional retrospectivo sobre una base de datos prospectiva desde el año 2014 hasta 2017. Se incluyeron de forma consecutiva pacientes con IAMCEST de $<12$ horas de evolución, que ingresaron para angioplastia primaria; $y$ se compararon según el período: pre- (2014-2015) o posimplementación de EIHM (2016-2017). Las ElHM evaluadas fueron: preactivación de hemodinamia y bypass de guardia. Como punto final primario se analizó el TPB. Como puntos secundarios se evaluó mortalidad a 180 días, reinfarto y nueva revascularización.

Resultados. Se analizaron 155 pacientes, 40,6\% dentro del grupo pre-EIHM y 59,4\% en el grupo post-EIHM. El 67\% llegó en ambulancia y el restante por sus propios medios. No existieron diferencias respecto al tratamiento médico instaurado (74\% recibió ticagrelor). Se observó una reducción del TPB en el grupo post-EIHM en comparación al preimplementación, de 42 min (rango intercuartílico $25-75$ [RIC]: 30-55,5) vs. 55 min (RIC: $32-77), p=0.014$, y en el tiempo total de isquemia, de 161,5 min (RIC: 120$239,5)$ vs. $197 \mathrm{~min}(145-277), p=0,02$. No se encontró diferencia significativa en mortalidad intrahospitalaria ( 3,1 vs. $2,1 \% ; p=0,7)$ ni en los eventos clínicos. El seguimiento se realizó en el 94,2\% de los pacientes, con una mediana de 7 meses (RIC: 1,23-15,7). A los 180 días no hubo diferencias en el punto final combinado (27,3 grupo pre- vs. $15,3 \%$ post-EIHM; log rank test $p=0,33$ ). En el modelo de regresión de Cox, la ElHM tuvo un hazard ratio de 0,69 (intervalo de confianza 95\%: 0,32-1,47 p=0,34).

Conclusión. Las EIHM implementadas lograron disminuir de forma significativa el TPB al igual que el tiempo total de isquemia, indicadores de la calidad del centro y del pronóstico intrahospitalario. Se observó una tendencia a la disminución del evento combinado en el seguimiento, aunque de forma no significativa.
\end{abstract}

Palabras clave: infarto del miocardio; infarto del miocardio con elevación del ST estrategias locales; mejoramiento de la calidad.

\section{ABSTRACT}

Introduction: The successful treatment of infarction with ST elevation (STEMI) is time dependent. There is limited information in our country about the effectiveness of strategies in reducing ischemia times. The aim of this work is to analyze the impact of the application of intrahospital strategies for improvement (IHSI) in the reduction of door-to-balloon time (DTB), and in the clinical evolution of patients with STEMI.

Methods: Retrospective observational study on a prospective database from 2014 to 2017. Patients with STEMI of $<12$ hours of evolution who were admitted for primary angioplasty were consecutively included; and they were compared according to the period: pre (2014-2015) or post implementation of IHSI (2016 2017). The evaluated IHSI were: preactivation of the hemodynamics lab and emergency bypass. The DTB was analyzed as the primary end point. As secondary points, 180 -day mortality, reinfarction and revascularization were evaluated. Results: 155 patients were analyzed, $40.6 \%$ in the pre-IHSI group and $59.4 \%$ in the post-IHSI group. $67 \%$ arrived by ambulance and the rest by their own means. There were no differences regarding the medical treatment established $(74 \%$ received ticagrelor). A reduction of DTB was observed in the post-IHSI group compared to the pre-implementation time; i.e. $42 \mathrm{~min}$ (PCTL 25-75: 30-55.5) vs. $55 \mathrm{~min}$ ( $P C T L$ 25-75: 32-77) $P=0.014$, and in the total ischemia time $161.5 \mathrm{~min}$ (PCTL 25 75 120-239.5) vs $197 \mathrm{~min}$ (145-277), $p=0.02$. No significant difference was found in in-hospital mortality (3.1 vs. $2.1 \%, p=0.7$ ) or in clinical events. Follow-up was performed in $94.2 \%$ of the patients, with a median of 7 months (PCTL 25-75: 1.23-15.7). At 180 days there were no differences in the composite end point (27.3 pre group vs. $15.3 \%$ post IHSI, log rank test $p=0.33$ ). In the Cox regression model, the IHSI had a hazard ratio of 0.69 ( $95 \%$ confidence interval $0.32-1.47 p=0.34$ ).

Conclusion: The implemented IHSI managed to significantly reduce the DTB, as well as the total time of ischemia, indicators of the quality of the center and the intrahospital prognosis. There was a tendency to decrease the composite event in the follow-up, although in a non-significant way.

Keywords: ST elevation; myocardial infarction; myocardial infarction; local strategies; quality Improvement.
1. Residente de Cardiología Clínica

2. Jefe de Residentes de Cardiología Clínica.

3. Jefe del Servicio de Cardiología Intervencionista y Terapéuticas Endovasculares.

4. Servicio de Cardiología Intervencionista y Terapéuticas Endovasculares. Instituto

Cardiovascular de Buenos Aires. Directora Iniciativa Stent - Save a Life. Argentina.

$\triangle$ Correspondencia: Dr. Alan Sigal. Blanco Encalada 1543, C1428DCO CABA, Rep. Argentina.alanrsigal@gmail.com.

Los autores declaran no tener conflictos de intereses.

Recibido: 14/10/2018| Aceptado: 01/11/2018

\section{INTRODUCCIÓN}

La enfermedad cardiovascular constituye la principal causa de muerte a nivel mundial, y en la Argentina no es una excepción, representando el 40\% ${ }^{1}$. La expresión más aguda de la cardiopatía isquémica es el infarto con elevación del segmento ST (IAMCEST).

La mortalidad en estos casos depende múltiples factores, pudiendo dividirse en los que involucran al paciente, como la edad, clasificación de Killip y Kimball y comorbilidades, y las que involucran a la atención médica recibida, principalmente el tiempo entre el inicio del dolor y la reperfusión?2.

El retraso del sistema, incluyendo la demora del paciente en consultar a servicios de emergencia, el tiempo en el traslado a centros con hemodinamia 
Tabla 1. Características basales. IMC: índice de masa corporal. ACV: accidente cerebrovascular. AHF: antecedentes heredofamiliares. ATC: angioplastía transcutánea. CRM: Cirugía de revascularización miocárdica. ElHM: estrategias intrahospitalarias de mejora. EPOC: enfermedad pulmonar obstructiva crónica. EVP: enfermedad vascular periférica. ERC: enfermedad renal crónica. HTA: hipertensión. IAM: infarto agudo de miocardio.

\begin{tabular}{|c|c|c|c|}
\hline & Pre-EIHM $(n=63)$ & Post-EIHM ( $n=92)$ & $\mathrm{p}$ \\
\hline Edad (media \pm DE, años) & $63,4 \pm 10,9$ & $62,8 \pm 12$ & 0,73 \\
\hline Sexo masculino $(n, \%)$ & $49(77,8 \%)$ & $22(23,9 \%)$ & 0,8 \\
\hline IMC (kg/m2) & $27,1 \pm 3,5$ & $27,3 \pm 3,8$ & 0,7 \\
\hline HTA $(n, \%)$ & $40(63,5 \%)$ & $60(65,2 \%)$ & 0,82 \\
\hline Diabetes (n, \%) & $8(12,7 \%)$ & $14(15,2 \%)$ & 0,65 \\
\hline Dislipemia (n, \%) & $30(47,6 \%)$ & $62(67,4 \%)$ & 0,014 \\
\hline Tabaquismo (n, \%) & $27(42,9 \%)$ & $26(28,3 \%)$ & 0,06 \\
\hline $\operatorname{AHF}(n, \%)$ & $14(22,2 \%)$ & $17(18,5 \%)$ & 0,56 \\
\hline EPOC $(n, \%)$ & $0(0 \%)$ & $4(4,3 \%)$ & 0,094 \\
\hline ACV previo (n, \%) & $1(1,6 \%)$ & $0(0 \%)$ & 0,22 \\
\hline EVP previa(n, \%) & $3(4,8 \%)$ & $6(6,5 \%)$ & 0,64 \\
\hline $\operatorname{ERC}(n, \%)$ & $0(0 \%)$ & $2(2,2 \%)$ & 0,23 \\
\hline IAM previo & $8(12,7 \%)$ & $16(17,4 \%)$ & 0,42 \\
\hline ATC previa & $9(14,3 \%)$ & $23(25 \%)$ & 0,1 \\
\hline CRM previa & $1(1,6 \%)$ & $4(4,3 \%)$ & 0,33 \\
\hline
\end{tabular}

y las demoras en derivación; es decir, todo el tiempo entre el inicio del dolor y la reperfusión adecuada, se asocia con mayor tamaño del infarto 3 y mayor mortalidad a corto y largo plazo ${ }^{24}$, siendo menor en los casos en los que se logra la reperfusión en menos de 60 minutos. Inclusive, se demostró que e tiempo entre la consulta y la activación del laboratorio de hemodinamia es un predictor independiente de mortalidad intrahospitalaria².

En este contexto, es fundamental diseñar e implementar estrategias a nivel institucional que logren disminuir los tiempos de cada centro, con el propósito de mejorar la sobrevida y evolución de los pacientes. Por lo tanto, el objetivo del presente trabajo fue analizar el impacto de la aplicación de estrategias intrahospitalarias de mejora (EIHM) en la reducción del tiempo puerta balón (TPB) y en la evolución clínica de pacientes con IAMCEST.

\section{MATERIALES Y MÉTODOS}

Se realizó un estudio observacional retrospectivo sobre una base de datos digitalizada cuya información fue recolectada en forma prospectiva desde el año 2014 hasta 2017 en el Instituto Cardiovascular de Buenos Aires (ICBA)

Se incluyeron pacientes mayores de 18 años con diagnóstico de IAMCEST de menos de 12 horas de evolución, que ingresaron traídos por ambulancia para angioplastia primaria (ATCP); y se compararon según el período: pre- (2014-2015) o posimplementación de EIHM (2016-2017).

Se definió IAMCEST acorde a la tercera definición universal de infarto y a las guías europeas de práctica clínica ${ }^{5,6}$. Se excluyeron pacientes que no cumplieran criterios de IAMCEST, con evolución mayor a 12 horas y aquellos que ingresaron derivados de otro centro.

En el año 2014 se implementaron dos ElHM: la preactivación de hemodinamia y el bypass de la guardia. Se estableció una red de comunicación entre servicios de emergencia médica que evaluaron pacientes en domicilio con dolor torácico y evidencia en electrocardiograma de supradesnivel del segmento ST y operadores entrenados en nuestro centro. Con el consenso diagnóstico de IAMCEST, se realizó la preactivación
Tabla 2. Tratamiento inicial. ElHM: estrategias intrahospitalarias de mejora.

$\begin{array}{lccc} & \text { Pre-EIHM }(n=63) & \text { Post-EIHM (n=92) } & p \\ \text { Prasugrel }(n, \%) & 1(1,6 \%) & 3(3,3 \%) & 0,51 \\ \text { Ticagrelor }(n, \%) & 49(77,8 \%) & 68(73,9 \%) & 0,58 \\ \text { Clopidogrel }(n, \%) & 10(15,9 \%) & 20(21,7 \%) & 0,36 \\ \text { Aspirina }(n, \%) & 60(95,2 \%) & 84(91,3 \%) & 0,34 \\ \text { Estatinas }(n, \%) & 54(85,7 \%) & 73(79,3 \%) & 0,22\end{array}$

Tabla 3. Resultados comparativos del punto final primario y del tiempo ECG-balón y total de isquemia. ECG: electrocardiograma. ElHM:estrategias intrahospitalarias de mejora.

\begin{tabular}{lccc} 
& Pre-EIHM $(\mathrm{n}=63)$ & Post-EIHM $(\mathrm{n}=92)$ & $\mathrm{p}$ \\
\hline $\begin{array}{l}\text { Tiempo total de isquemia } \\
\text { (mediana; RIC) }\end{array}$ & $197(145-277)$ & $161.5(120-239.5)$ & 0,02 \\
$\begin{array}{l}\text { Tiempo ECG-balón } \\
\text { (mediana; RIC) }\end{array}$ & $85(61-117)$ & $70(41-108,5)$ & 0.012 \\
$\begin{array}{l}\text { Tiempo puerta-balón } \\
\text { (mediana; RIC) }\end{array}$ & $55(32-77)$ & $42(30-55,5)$ & 0,014
\end{tabular}

de hemodinamia consistente en la llegada de médicos intervencionistas y preparación de la sala previo al arribo del paciente. Y posteriormente se implementó el bypass de la guardia, consistente en el ingreso directo del paciente a la sala sin previa evaluación por guardia de emergencias. El seguimiento fue realizado mediante controles ambulatorios y de forma telefónica a los 30,60 y 180 días del alta.

Como punto final primario se analizó el TPB. Como puntos secundarios se evaluó mortalidad a 180 días, reinfarto y nueva revascularización coronaria.

\section{ANÁLISIS ESTADÍSTICO}

Las variables categóricas se expresaron mediante números y porcentajes, y las continuas, según su distribución, como media o mediana, con su correspondiente desvío estándar o rango intercuartílico (RIC). Para evaluar la asociación entre las variables continuas se utilizó el test de U de Mann-Whitney o test de T, y para las categóricas se utilizó el test exacto de Fisher o Chi Cuadrado, según correspondiera. El análisis de sobrevida entre ambos subgrupos se construyó utilizando el método de Kaplan-Meier y se comparó mediante la prueba de log rank, y se realizó un modelo de regresión múltiple por método de Cox, expresado como hazard ratio (HR) y su intervalo de confianza de 95\% (IC95\%). Se incluyeron en el modelo las variables que presentaron una $p<0,05$ en el análisis univariado. Se consideró estadísticamente significativo un valor $p$ a dos colas menor o igual a 0,05. Para el análisis estadístico se utilizó el software SPSS 23.

\section{CONSIDERACIONES ÉTICAS}

El estudio fue conducido de acuerdo con las normas éticas nacionales (ley CABA 3301) y Ley nacional de investigación clínica en seres humanos, declaración de Helsinki y contó con la aprobación del Comité de Investigación y Ética de nuestra Institución.

\section{RESULTADOS}

Se incluyeron 155 pacientes, 63 en el primer grupo y 92 en el segundo. El promedio de edad entre los dos grupos fue de 63,1 años; $46 \%$ eran de sexo masculino; $64 \%$ hipertensos, $13,5 \%$ diabéticos, 35\% tabaquistas. No se encontraron diferencias significativas en las características basales de la población (Tabla 1), a excepción de que se encontraron más pacientes con dislipemia en el grupo post-EIHM 
Tabla 4. Objetivos secundarios de evolución intrahospitalaria. FEVI: fracción de eyección del ventrículo izquierdo. ATC: angioplastia transluminal coronaria. DES: stent liberador de drogas. IRA: insuficiencia renal aguda. PCR: paro cardiorrespiratorio. ElHM: estrategias intrahospitalarias de mejora.

\begin{tabular}{lccc} 
& $\begin{array}{c}\text { Pre-EIHM } \\
(\mathrm{n}=63)\end{array}$ & $\begin{array}{c}\text { Post-EIHM } \\
(\mathrm{n}=92)\end{array}$ & $\mathrm{P}$ \\
\hline Killip y Kimball $(\mathrm{n}, \%)$ & $52(82.5 \%)$ & $75(81,5 \%)$ & 0,9 \\
\hline IV & $2(3,1 \%)$ & $4(4,3 \%)$ & \\
\hline FEVI \% (mediana; RIC) & $55(32-77)$ & $50(45-56)$ & 0,014 \\
\hline Revascularización completa $(\mathrm{n}, \%)$ & $17(26,9 \%)$ & $21(22,8 \%)$ & 0,20 \\
\hline ATC con DES (n, \%) & $32(50,7 \%)$ & $83(90,2 \%)$ & $<0.001$ \\
\hline Sangrado TIMI mayor (n, \%) & $4(6,3 \%)$ & $7(7,6 \%)$ & 0,9 \\
\hline Complic. accesos vasculares $(n, \%)$ & $10(15,8 \%)$ & $8(8,6 \%)$ & 0,17 \\
\hline IRA (n, \%) & $2(3,1 \%)$ & $4(4,3 \%)$ & 0,7 \\
\hline Complicaciones mecánicas $(n, \%)$ & $2(3,1 \%)$ & $2(2,1 \%)$ & 0,7 \\
\hline Shock cardiogénico (n, \%) & $6(9,5 \%)$ & $6(6,5 \%)$ & 0,49 \\
\hline PCR & $2(3,1 \%)$ & $8(8,6 \%)$ & 0,16 \\
\hline Muerte (n, \%) & $2(3,1 \%)$ & $2(2,1 \%)$ & 0,7 \\
\hline Arritmias ventriculares $(n, \%)$ & $8(12,6 \%)$ & $11(11,9 \%)$ & 0,8 \\
\hline
\end{tabular}

$(67,4 \%)$ que en el grupo preintervención $(47,6 \%)(p=0,014)$. El 15\% de pacientes presentó antecedentes de cardiopatía isquémica.

No se evidenciaron diferencias en el tratamiento farmacológico instaurado en ambos grupos (Tabla 2). La mayoría de los pacientes fueron tratados con ticagrelor como segundo antiagregante $(75,8 \%)$, mientras que el clopidogrel fue el segundo más elegido (18,8\%). El 93\% de los pacientes recibió tratamiento con aspirina y el 82,5\% con estatinas.

En cuanto al punto final primario, el TPB fue más corto en el grupo en el cual se implementaron las estrategias de mejora intrahospitalaria de forma estadísticamente significativa, con una reducción de la mediana de 13 minutos (55 minutos vs. 42 minutos; $p=0,014$ ). Además, el tiempo ECG-balón ( 85 minutos vs. 70 minutos; $p=0,012$ ) y el tiempo total de isquemia (197 minutos vs. 161,5 minutos; $p=0,02$ ) también se redujeron de forma significativa (Tabla 3).

En cuanto a los objetivos secundarios de evolución intrahospitalaria, no se encontraron diferencias en cuanto a insuficiencia renal aguda, complicaciones mecánicas, arritmias ventriculares o muerte. Hubo mayor tasa de uso de DES en el grupo posimplentación de las ElHM que en el grupo preimplementación (90,2 vs. 50,7\%; $p<0,001)$ (Tabla 4).

El seguimiento se realizó en el 94,19\% de los pacientes, con una mediana de 7 meses (RIC: 1,23-15,7). A los 180 días no hubo diferencias en el punto final combinado de mortalidad, reinfarto y nueva revascularización, ocurriendo en un 27,3\% de pacientes en el grupo pre- y 15,3\% en el grupo post-EIHM (log rank test $\mathrm{p}=0,33$ ). Al evaluar la asociación entre estas medidas y la supervivencia en el seguimiento mediante un modelo de regresión de Cox, se obtuvo un HR de 0,69 (IC95\%: 0,32-1,47; p=0,34) (Figura 1).

\section{DISCUSIÓN}

En este estudio encontramos que la implementación del bypass de la guardia y la preactivación de hemodinamia en pacientes con IAMCEST se asoció a una reducción del tiempo puerta-balón. También se observó una reducción en el tiempo total de isquemia, y una tendencia a la reducción del endpoint secundario combinado de eventos clínicos que no alcanzó significancia estadística.

Según una evaluación realizada por la Organización Mundial de la
Salud y la Organización Panamericana de la Salud', en nuestro país la muerte cardiovascular representa el $29 \%$ de la mortalidad prematura (entre 30 a 69 años) en hombres y el 23\% en mujeres. El 31\% de estas muertes en hombres, y $21 \%$ en mujeres, fueron de causa isquémica. En Argentina, esta entidad tiene una mortalidad intrahospitalaria de 8,8\% en promedio según el mayor y último registro realizado a nivel nacional, el Argen-IAM-ST de 2015, similar a los valores obtenido en el SCAR de 2011, con una mortalidad del 6,2\%, y al registro CONAREC de 2009, con 8,2\%,8. Considerando registros históricos en nuestro país, como el SCAR 2005 en el cual la mortalidad hospitalaria fue de 13,8\%, la mortalidad relacionada al IAMCEST ha disminuido con el desarrollo de técnicas de reperfusión; pero sigue siendo elevada en comparación con registros internacionales (de 4 a $6 \%$ en algunos países europeos ${ }^{9}$ ).

Durante un IAMCEST, hay 3 marcadores principales que definen la magnitud del daño miocárdico: la localización de la lesión (que determinará la cantidad de miocardio en riesgo), el flujo colateral y el tiempo de reperfusión ${ }^{10}$. Otro estudio, ya en el 2004, demostró que por cada 30 minutos de retraso en la angioplastia aumenta 7.5\% la mortalidad ${ }^{11}$, y otro estudio de 30.000 pacientes del 2006 demostró una mortalidad del 3\% con TPB menor a 90 minutos, comparado con 4,2, 5,7 y 7,4 para tiempos de 90-120 minutos, 91-150 minutos y más de 150 minutos, respectivamente ${ }^{12}$.

De todas maneras, el impacto de los retrasos en la reperfusión no es igual para todos los pacientes: un registro de más de 1800 pacientes de Estados Unidos ${ }^{13}$ demostró un aumento progresivo de la mortalidad con el retraso en reperfusión en los pacientes con shock cardiogénico, mientras que en aquellos sin shock la mortalidad se mantuvo sin cambios significativos hasta las 6 horas. En este contexto, las medidas que disminuyan los tiempos de reperfusión tienen potencial de disminuir aún más el tamaño del infarto y los eventos a corto y largo plazo, sobre todo en aquellos pacientes de mayor riesgo.

En cuanto a los tiempos de reperfusión en Argentina, acorde al ya mencionado Argen-IAM-ST 14, la demora entre el inicio del dolor y la consulta médica tuvo una mediana de 170 minutos. La mediana de tiempo puerta-balón fue de 95 minutos, mientras que menos de la mitad de los pacientes tuvieron un tiempo menor a 90 minutos como indican las guías de práctica clínica. El tiempo total de isquemia tuvo una mediana de 300 minutos.

En nuestro país, se encuentra publicada una serie de Mendoza ${ }^{15}$ del año 2016, en el cual se registraron los tiempos de reperfusión de IAMCEST en un centro de dicha provincia, dividiéndolos en 5 etapas: consulta, traslado, guardia, unidad coronaria y hemodinamia. Allí identificaron que el tiempo hasta la consulta representó el 40-50\% de los retrasos, y que el tiempo entre la consulta y la decisión terapéutica representó un $35 \%$ del tiempo si se trataba de un centro periférico derivador y $15 \%$ si fue en el hospital. La mediana de tiempo total de isquemia en dicha serie fue de 303 minutos, y del tiempo puerta-balón de 107 minutos. En el registro CathPCl de Estados Unidos, que abarca más de 1500 hospitales a lo largo de todo el país, con más de 600.000 pacientes incluidos en el 2014, un 93,5\% de los pacientes que consultaron a un centro con capacidad de ATCp tuvieron un tiempo puerta-balón menor a 90 minutos, mientras que en aquellos derivados desde un centro sin hemodinamia solo un 32,3\% alcanzaron este objetivo ${ }^{16}$.

Otro estudio de Estados Unidos demostró que dentro de 28 posibles 


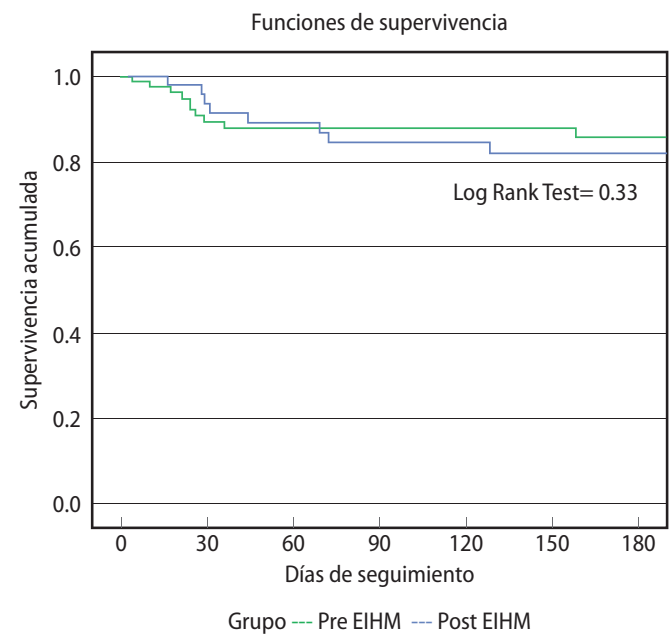

Figura 1. Curvas de supervivencia para punto final combinado de mortalidad, reinfarto y nueva revascularización. ElHM: estrategias intrahospitalarias de mejora.

estrategias para disminuir estos tiempos, 6 se asociaron de forma significativa a una reducción eficaz ${ }^{17}$. Estas fueron: preactivación de hemodinamia por staff de emergencias, preactivación a través de un único llamado a un operador central, preparación de la sala de hemodinamia mientras el paciente se encuentra en camino al centro, llegada de hemodinamistas en menos de 20 minutos tras el llamado, tener un cardiólogo 24 hs en el centro, y el uso de feedback en tiempo real entre la sala de emergencias y la de hemodinamia.

Desde el años 2015 la Argentina está participando de la Iniciativa Stent-Save a Life ${ }^{18}$, programa creado por la Sociedad Europea de Cardiología, el EuroPCR y la Asociación Europea de Intervenciones Cardiovasculares Percutáneas (EAPCI) cuya misión es mejorar el acceso de los pacientes con IAMCEST a un tratamiento de reperfusión de calidad y dentro de los tiempos recomendados por las guías clínicas El ICBA es uno de los 45 centros del país que se encuentran formando parte de su programa de mejora de tiempos puerta-balón.

En cuanto a las limitaciones de nuestro trabajo, al ser un estudio retrospectivo no calculamos la potencia de nuestra muestra para nuestro punto final primario. Los puntos finales secundarios deben además tomarse como exploratorios, dado que no podemos establecer causalidad directa ni contamos con la cantidad de pacientes necesarios para evaluarlo correctamente. También debemos tener en cuenta que existen muchos factores no evaluados que también pueden influir sobre los tiempos puerta-balón y total de isquemia en los años estudiados, como por ejemplo diferentes realidades del país en cuanto a redes de ambulancias. Al estar en un centro monovalente de alta complejidad con hemodinamia las 24 hs, es posible que nuestros tiempos no reflejen la realidad del país. Muy probablemente los retrasos en reperfusión de nuestra institución obedecen principalmente a cuestiones internas, mientras que en otros centros respondan más a las dificultades extrahospitalarias. Para poder implementar estas estrategias a nivel país, es necesario contar con redes de atención, con conexión fluida entre ambulancias, centros con hemodinamia y centros sin hemodinamia y con protocolos estrictos que logren que se pierda el menor tiempo posible, y así poder disminuir los eventos de nuestros pacientes.

\section{CONCLUSIÓN}

En este estudio observacional retrospectivo unicéntrico, la implementación de estrategias intrahospitalarias de mejora, como la preactivación de hemodinamia y el bypass de la guardia, se asociaron de forma significativa con la reducción de los tiempos de reperfusión en el IAMCEST, con una tendencia a la reducción de eventos cardiovasculares mayores.

\section{BIBLIOGRAFÍA}

1. OPS/OMS, Situación de Salud en las Américas: Indicadores Básicos 2013. Washington, D.C. Estados Unidos de América, 2013.

2. Terkensen CJ, Sørensen JT, Maeng M, Jensen LO, Tilsted HH, Trautner S, et al. Sys tem delay and mortality among patients with STEMI treated with primary percutaneous coronary intervention JAMA 2010:304(7):763-771.

3. Guerchicoff A, Brener SJ, Maehara A, Witzenbichler B, Fahy M, Xu K, et al. Impact of Delay to Reperfusion on Reperfusion Success, Infarct Size, and Clinical Outcomes in Patients With ST-Segment Elevation Myocardial Infarction: The INFUSE-AMI Trial (INFUSE-Anterior Myocardial Infarction). J Am Coll Cardiol Int 2014;7(7):733-740.

4. Lambert L, Brown K, Segal E, Brophy J, Rodes-Cabau J, Bogaty P. Association Between Timeliness of Reperfusion Therapy and Clinical Outcomes in ST-Elevation Myocardial Infarction. JAMA 2010;303(21):2148-2155.

5. Thygesen K, Alpert JS, Jaffe AS, Simoons ML, Chaitman BR, White HD, et al. Third Universal Definition of Myocardial Infarction. Eur Heart J 2012; 33(20):2551-2567

6. Ibanez B, James S, Agewall S, Antunes MJ, Bucciarelli-Ducci C, Bueno H, et al. 2017 ESC Guidelines for the management of acute myocardial infarction in patients presenting with ST-segment elevation: The Task Force for the management of acute myocardial infarction in patients presenting with ST-segment elevation of the European Society of Cardiology (ESC). Eur Heart J 2018;39(2):119-177

7. Higa C. Comparación temporal de dos registros multicéntricos argentinos de infarto con elevación del segmento ST: SCAR 2011 - ARGEN IAM ST 2015. Presentation presented at; $2016 ; 42^{\circ}$ Congreso Argentino de Cardiología.

8. Pérez GE, Costabel JP, González N, Zaidel E, Altamirano M, Schiavone M et al. Infarto agudo de miocardio en la República Argentina: Registro CONAREC XVII. Rev Argen Cardiol 2013;81(5):390-399.

9. Kristensen SD, Laut KG, Fajadet J, Kaifoszova Z, Kala P, Di Mario C, et al. Reperfusion therapy for ST elevation acute myocardial infarction 2010/2011: current status in 37 ESC countries. Eur Heart J 2014;35(29):1957-70.

10. Christian TF, Schwartz RS, Gibbons RJ. Determinants of infarct size in reperfusion therapy for acute myocardial infarction. Circulation 1992:86(1):81-90.

11. De Luca G, Suryapranata H, Ottervanger JP, Antman EM. Time delay to treatment and mortality in primary angioplasty for acute myocardial infarction: every min ute of delay counts. Circulation 2004:109(10):1223-1225.

12. McNamara RL, Wang Y, Herrin J, Curtis JP, Bradley EH, Magid DJ, et al. Effect of Door-to-Balloon Time on Mortality in Patients With ST-Segment Elevation Myo cardial Infarction. J Am Coll Cardiol 2006:47(11):2180-6

13. Brodie BR, Stuckey TD, Muncy DB, Hansen CJ, Wall TC, Pulsipher M, et al. Importance of time-to-reperfusion in patients with acute myocardial infarction with and without cardiogenic shock treated with primary percutaneous coronary intervention. Am Heart I 2003:145(4):708-715.

14. Gagliardi JA, Charask A, Perna E, D'imperio H, Bono J, Castillo Costa Y, et al. Encuesta Nacional de infarto agudo de miocardio con elevación del ST en la República Argentina (ARGENT-IAM-ST). Rev Fed Arg Cardiol 2017;46(1):15-21

15. Ortego J, Aveni S, Ripa L, Estrella N, Suriano J, Ferraro M, et al. Análisis de tiempos de intento de reperfusión en pacientes con infarto con elevación de ST. Revista CONAREC. 2016:32(137):274-278.

16. Masoudi FA, Ponirakis A, de Lemos JA, Jollis JG, Kremers M, Messenger JC, et al. Trends in U.S. Cardiovascular Care: 2016 Report From 4 ACC National Cardiovascular Data Registries, J Am Coll Cardiol. 2017:69(11):1427-1450.

17. Bradley EH, Herrin J, Wang Y, Barton BA, Webster TR, Mattera JA, et al. Strategies for Reducing the Door-to-Balloon Time in Acute Myocardial Infarction. N Engl Med 2006:355(22):2308-2320

18. Iniciativa Stent - Save a Life! Argentina. Disponible en http://stentsavealife.com ar. Consultado el 20/11/2018. 